\title{
Piston-cylinder apparatus for high-pressure impedance spectroscopy
}

Cite as: Review of Scientific Instruments 68, 193 (1997); https://doi.org/10.1063/1.1147808

Submitted: 17 July 1996 . Accepted: 17 September 1996 . Published Online: 04 June 1998

R. Hinrichs, and J. A. H. da Jornada

ARTICLES YOU MAY BE INTERESTED IN

Short piston-cylinder pressure cells based on $\mathrm{Ni}-\mathrm{Cr}-\mathrm{Al}$ cylinders and their application to fragile materials

Review of Scientific Instruments 81, 033903 (2010); https://doi.org/10.1063/1.3310197

Creating a high temperature environment at high pressure in a gas piston cylinder apparatus

Review of Scientific Instruments 83, 014501 (2012); https://doi.org/10.1063/1.3677844

Nonmagnetic piston-cylinder pressure cell for use at $35 \mathrm{kbar}$ and above

Review of Scientific Instruments 70, 3402 (1999); https://doi.org/10.1063/1.1149927

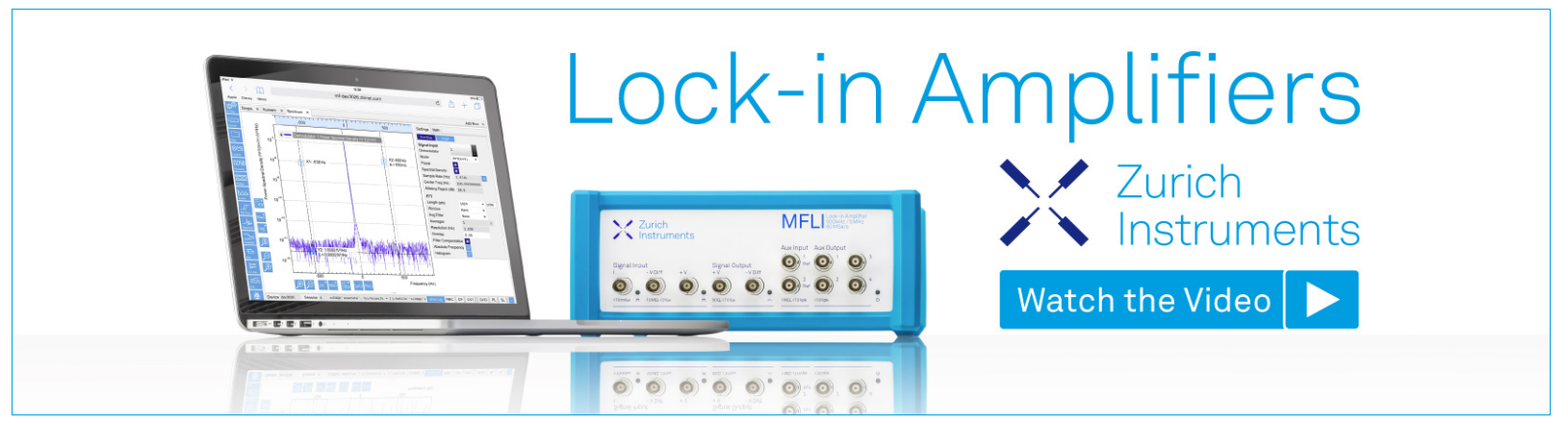

Review of Scientific Instruments 68, 193 (1997); https://doi.org/10.1063/1.1147808 


\title{
Piston-cylinder apparatus for high-pressure impedance spectroscopy
}

\author{
R. Hinrichs ${ }^{a)}$ \\ Institut für Werkstoffwissenschaften 3, FAU, Martensstrasse 5, 91018 Erlangen, Germany \\ J. A. H. da Jornada \\ Instituto de Física UFRGS, Av. Bento Gonçalves 9500, 91501 Porto Alegre, RS, Brazil
}

(Received 17 July 1996; accepted for publication 17 September 1996)

\begin{abstract}
A simple piston-cylinder apparatus was developed for impedance spectroscopy at pressures up to $3 \mathrm{GPa}$ and temperatures up to $500{ }^{\circ} \mathrm{C}$. The apparatus is of the compound cylinder type, with the innermost cylinder made of a commercially available low-cost alumina-based ceramic, with pistons of hard metal that allow electric connections. Cell electrical resistance was higher than $1 \mathrm{M} \Omega$ at $T<300{ }^{\circ} \mathrm{C}$ and higher than $100 \mathrm{k} \Omega$ at $400{ }^{\circ} \mathrm{C}$. The inductivity in the wires and pistons was about 2 $\mu \mathrm{H}$. The sample was enclosed in a $h$-BN capsule, which insured a good hydrostaticity without disturbing the measurement. The equipment proved to be very useful in determining the activation volume of the conduction process in several ionic conductors. (C) 1997 American Institute of Physics. [S0034-6748(96)03012-2]
\end{abstract}

\section{INTRODUCTION}

Ionic conductivity is usually measured with ac methods to prevent electrode polarization, since the electrical carriers inside the ion conducting materials are of a different kind than in the outer circuit. However, it is not enough to obtain ac measurements at a fixed frequency, as has been done in some pressure-dependence resistivity reports. The impedance of a real material is a sum of partial impedances $\left(Z^{*}\right)$ that are complex functions of frequency as can be seen in Eq. (1):

$$
Z^{*}(\omega)=Z_{\infty}+\frac{Z_{0}-Z_{\infty}}{1+(i \omega \tau)^{1-\alpha}},
$$

where $Z_{0}$ is the zero-frequency (dc) resistance, $Z_{\infty}$ is the high-frequency limit of the impedance (usually zero), $\tau$ is the relaxation time, and $\alpha$ a non-Debye factor $(0<\alpha<1)$. ${ }^{1}$ Since relaxation time is pressure dependent, fixed-frequency measurements will introduce serious errors in the resistivity determination. Therefore, it has become routine in ionics to use impedance spectroscopy (IS), namely, the measurement of ac complex resistance over a broad range of frequencies. The conductivity is determined from the spectra afterwards, with the aid of complex nonlinear least-square fitting procedures. This technique is of greatest importance in the study of polycrystalline samples, where grain and grain-boundary conductivities are simultaneously present and cannot be isolated experimentally. Since different dielectric dispersion processes in grains and grain boundaries have different relaxation times, it is usually possible to determine the bulk properties without the need of a single crystal.

Usually the impedance spectra are displayed in complex-plane plots (root loci), with the imaginary part of the impedance plotted against the real part $[-\operatorname{Im}(Z)$ against $\operatorname{Re}(Z)$ ]. Arcs will become visible in these plots that are related to regions of different electric response (bulk, grain boundary, electrodes), depending on typical capacitance val-

${ }^{a)}$ Present address: Instituto de Física UFRGS, c.p. 15051, 91501-970 Porto Alegre, RS, Brazil; Electronic mail: ruthh@if.ufrgs.br ues. Bulk conductivity can be determined if the measured frequency range contains the inverse of bulk relaxation time, so that the complex-plane plot contains the maximum of the bulk impedance arc (for an excellent review of IS see Ref. 1).

The study of the pressure dependence of ionic conductivity allows the determination of the activation volume of the conduction process and produces insights on the mechanisms involved in ion conduction. ${ }^{2}$ To obtain the pressure dependence of conductivity, it is necessary to measure the impedance of the sample in a pressurized container. This pressure chamber should be conceived in a way to minimize parasitic impedance, resulting from the connecting wire inductance and from leak currents in the chamber walls.

This paper describes a simple apparatus, that allows rapid IS measurements of medium-sized samples under moderate temperature and pressure. It was successfully used to determine the temperature and pressure dependence of the bulk conductivity of some ionic conductors $\left(\mathrm{NH}_{4} \mathrm{NbWO}_{6},{ }^{3,4} \mathrm{SrCeO}_{3},{ }^{4} \mathrm{AgBr},{ }^{4}\right.$ polyaniline salts $\left.{ }^{5}\right)$. From these data it was possible to establish the conductance activation volume and activation energy in the temperature range $20-400{ }^{\circ} \mathrm{C}$ and pressure range $0-3 \mathrm{GPa}$.

\section{DESCRIPTION OF THE HIGH-PRESSURE CHAMBER}

The IS high-pressure chamber was based on a compound-cylinder geometry, with the distinction, that the innermost cylinder was not made of hard metal, as usual, but was made of a high-strength ceramic. The ceramic was a commercially available high-density hot-pressed aluminazirconia cutting insert (Cerasiv SN80). This material offered all the properties required, as high hardness and toughness, low electrical conductivity, good thermal conductivity, as well as low cost.

The basic structure of the chamber is shown in Fig. 1. The main part was a ceramic cylinder with a central sample room, that was surrounded by three supporting rings made of hardened steel. This compound cylinder was enclosed in a steel mantle mounted with screws that contained the pistons 


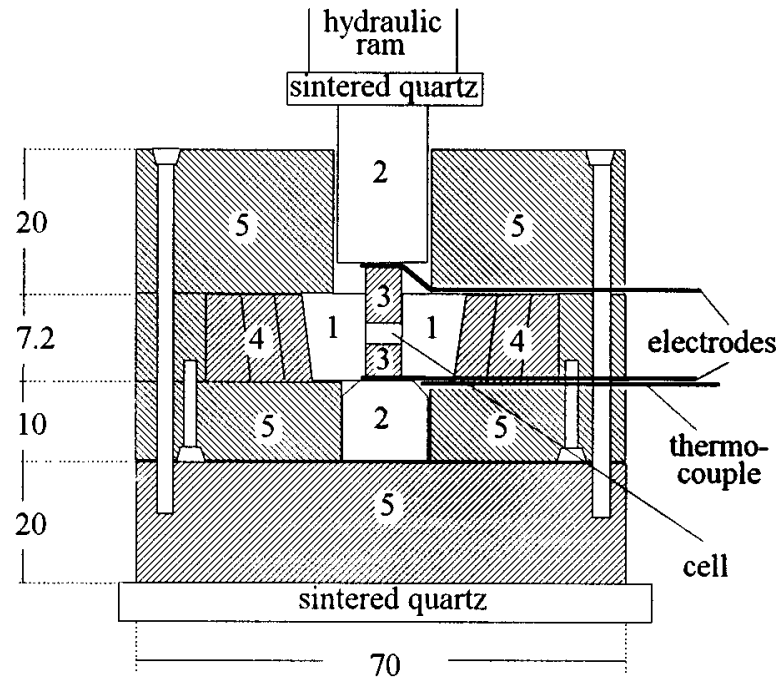

FIG. 1. High pressure chamber with: 1-ceramic ring, 2-insulating and 3-conducting pistons, 4-hardened steel rings, 5-steel mantle (dimensions in $\mathrm{mm}$ ).

and gave them lateral guidance. Two stages of pistons transmitted the pressure to the sample. The outer stage consisted of high strength ceramic pistons made of Sialon (for our workshops convenience. They could have been made of alumina as well). The pistons of the inner stage were made of hard metal (Hertel micrograin quality with $5 \%$ Co binder). The driving force for the pistons was generated by an external 3 ton press.

In order to obtain high temperature at the sample, the whole chamber was heated from the outside with the aid of a tightly fitting copper belt, that contained six $150 \mathrm{~W}$ heating elements and was controlled by an external heating controller. Sintered quartz blocks were placed between the outer pistons and the hydraulic ram to reduce thermal flow to the press.

The construction details of the compound cylinder are shown shematically in Fig. 2. The concentric steel rings (maraging steel hardened to about $50 \mathrm{RcC}$ ) with taper angle of $1^{\circ}$ were forced into each other with interference. The interferences were calculated to increase the strength of the inner

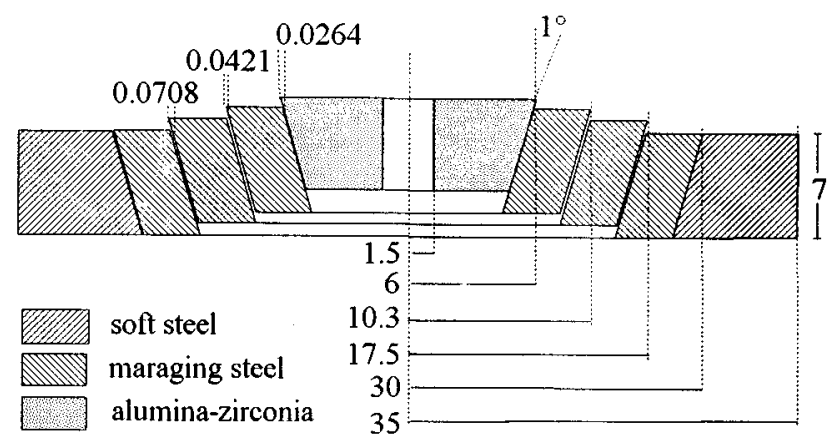

FIG. 2. Schematic view of the compound cylinder before assembling, showing the interferences (dimensions in $\mathrm{mm}$ ).

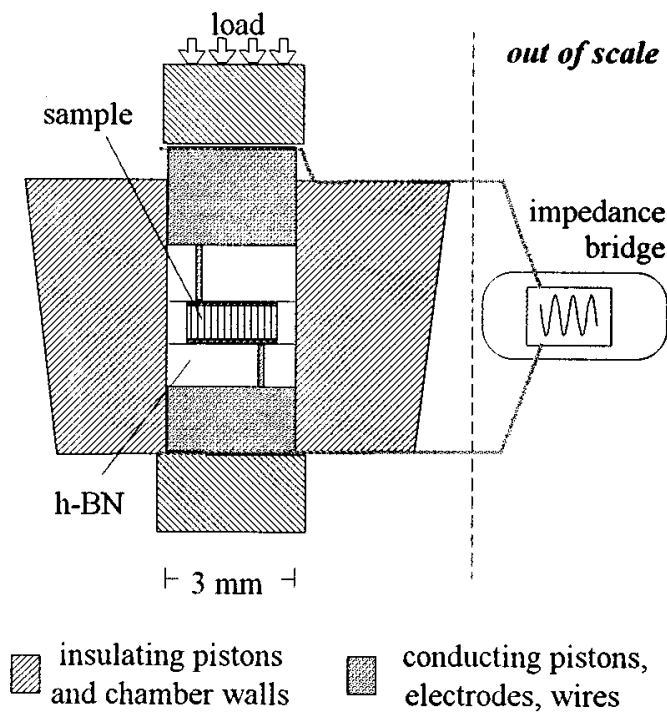

FIG. 3. High pressure core of the cell with electrical contacts to the impedance bridge.

ceramic ring, compensating the different thermal expansions of the ceramic and the metallic rings and producing a high contact pressure in the temperature range from RT to $500{ }^{\circ} \mathrm{C}$ (figures are displayed in Fig. 2). This assembly was encased in a soft steel belt, designed to prevent damage to the operator in the event of accidental breaking of the rings. The ceramic part was carefully pressed into the compound steel ring. The central hole was finished with diamond paste after assembling.

\section{CELL CONFIGURATION}

The selected pressure transmitting medium was highdensity sintered hexagonal boron nitride $(h$-BN), because of its low shear strength, that gave good hydrostaticity. This material also had the other characteristics required for the experiments, namely, a very high electrical resistivity, low compressibility, and an excellent chemical inertness. Furthermore, it could be easily machined to rings and platelets.

The cell configuration can be seen in Fig. 3. The pressed powder or sintered sample pellets were inserted in machined $h$-BN rings and covered on top and bottom with gold foil (internal electrodes) and two thin $h$-BN pellets. Small excentric copper feedthroughs made the electric contact between the hard-metal pistons and the internal electrodes. External electrodes were obtained squeezing a $5 \mu \mathrm{m}$ copper foil between the insulating and the conducting hard-metal pistons. These copper platelets were spot welded to $0.2 \mathrm{~mm}$ copper wires, insulated with alumina tubes and brought to the outside through slots in the steel mantle. The wires were connected to the coaxial cables of the impedance bridge, allowing the application of an ac electric field on the internal pistons and the sample between them. A thermocouple insulated with a double-hole alumina tube was inserted through the same slot. 


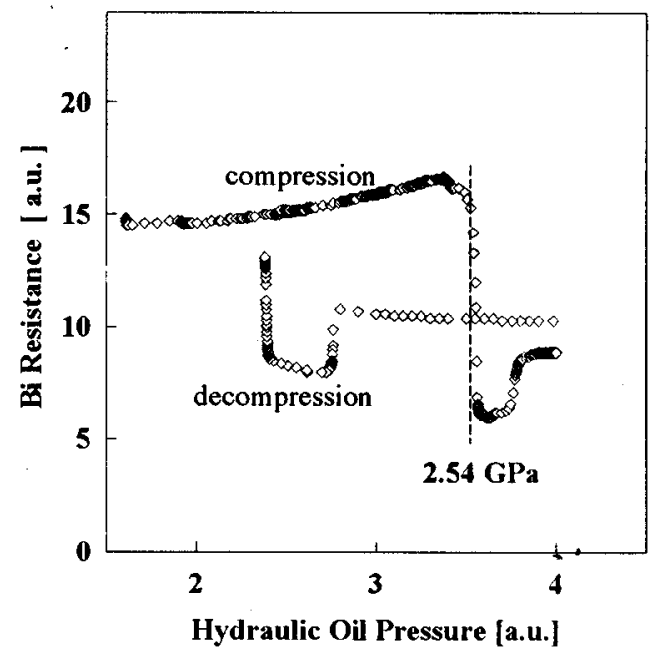

FIG. 4. Pressure calibration with the transition Bi I-Bi II at $2.54 \mathrm{GPa}$.

\section{RESULTS}

The pressure in the chamber was determined by measuring press force and correcting this value for losses due to mechanical friction and internal friction in the pressure transmitting medium. A calibration with the well-known pressure induced transition point of Bi I-BI II (Ref. 6) is shown in Fig. 4. The resistance of $\mathrm{Bi}$ against pressure on compression and decompression in an embedding of $h$-BN showed that the value expected on the geometrical relation of the areas of piston and hydraulic ram had to be corrected subtracting $15 \%$ for losses. The compression cycle shows a sharp decrease in the resistivity at the transition point, indicating good hydrostaticity. On decompression there is a significant hysteresis. To overcome internal friction and pressure gradients in the pressure transmitting medium and to get an equilibrium inside the chamber, the pressure was cycled around the desired nominal value by successively decreasing steps.

To gather information about the parasitic electrical behavior of the chamber at high temperatures, the sample space was filled with $h$-BN and the impedance was measured at various temperatures and pressures. The conductance of the assembly is shown in Fig. 5 as a function of temperature and pressure. At room temperature, the conductance is less than $10^{-7} \mathrm{~S}$ and rises three orders of magnitude while heating to $500{ }^{\circ} \mathrm{C}$ due to the increasing conductivity of the aluminazirconia walls. At pressures higher than 0.5 GPa temperature dependence is much smaller, showing an increase of only one decade in this temperature range. These conductance values are very low compared to the ion-conducting materials usually studied under pressure, so the effect of parallel conduction through the chamber walls could be generally disregarded.

In order to evaluate the performance of this equipment, the ion conductivity of silver bromide was measured. At temperatures below $100{ }^{\circ} \mathrm{C}$, the values we obtained for the conductivity of $\mathrm{AgBr}$ and the calculated activation volume for $\mathrm{Ag}^{+}$transport $\left(5.1 \pm 0.9 \mathrm{~cm}^{3} / \mathrm{mol}\right)$ shows satisfactory agreement with the data of Lansiart et al., ${ }^{7}$ which report an

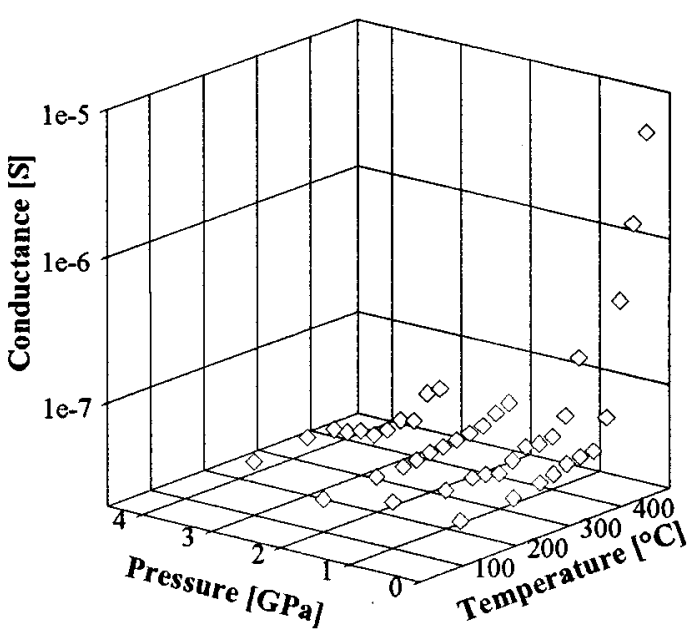

FIG. 5. Conductance behavior of the chamber assembly at different temperatures and pressures.

activation volume of $5.5 \pm 0.5 \mathrm{~cm}^{3} / \mathrm{mol}$ measured under hydrostatic argon pressure up to $0.8 \mathrm{GPa}$.

The chamber was originally designed to study the ionconducting properties of pressed powder samples of the pyrochlore $\mathrm{NH}_{4} \mathrm{NbWO}_{6}{ }^{3,4}$ In Fig. 6 the complex-plane plots of two impedance spectra, taken at $130{ }^{\circ} \mathrm{C}$ and pressures of 0.04 and $0.45 \mathrm{GPa}$ are shown. The grain (left) and grainboundary (right) impedance arcs can be perceived clearly.

When the chamber was used to measure pressure dependence of highly conductive polymers, ${ }^{5}$ with impedance values typically in the range of $10-100 \Omega$, the inductivity due to the connecting wires became important. Calibration of the short-circuited chamber showed a value of $2 \mu \mathrm{H}$, which became a significant contribution at frequencies above $1 \mathrm{MHz}$. This inductivity had to be included as an additional parameter in the least-square fitting procedure, because it caused a depression of the measurement points at high frequencies. Figure 7 shows two complex-plane plots of a conductive polyaniline salt at different pressures $(0.08$ and $0.4 \mathrm{GPa}$, the measurement points are the crosses, the inductivity-corrected

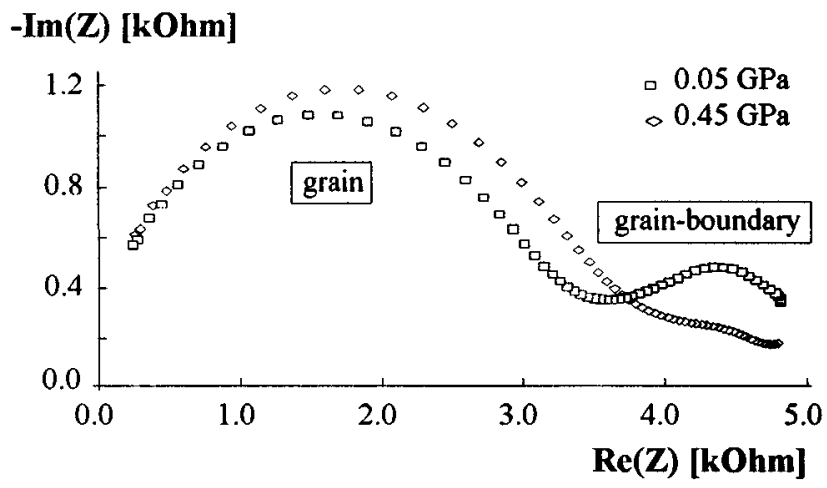

FIG. 6. Complex-plane plots of $\mathrm{NH}_{4} \mathrm{NbWO}_{6}$ measured at $130{ }^{\circ} \mathrm{C}$ and 0.05 and $0.45 \mathrm{GPa}$. 


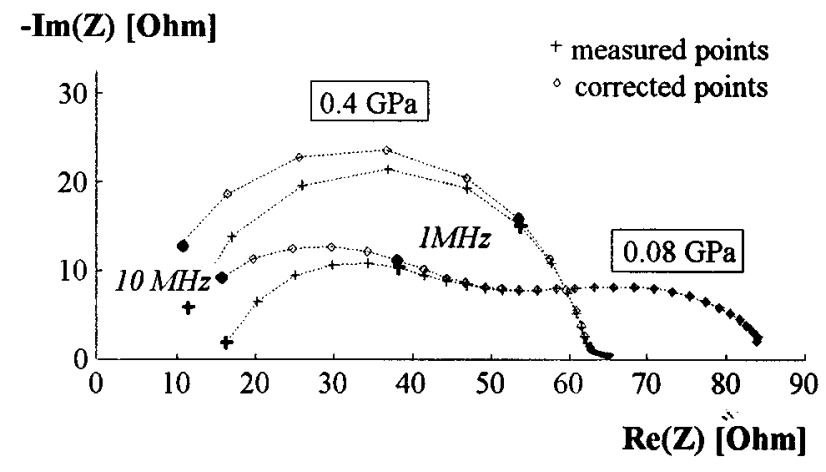

FIG. 7. Impedance of polyaniline salts at different pressures and the correction for parasitic impedance of the chamber (bold points correspond to 10 $\mathrm{MHz}$, every five points correspond to one frequency decade).

points are the diamonds). It can be seen that only in the high frequency region the inductivity correction is significant.

\section{DISCUSSION}

A low-cost high-pressure apparatus suitable for impedance measurements of polycrystalline ion conducting samples was developed. The apparatus was designed to be used in a small press and allowed sample diameters of 1.8 $\mathrm{mm}$. This size allows easy handling of the pressed-powder or sintered sample pellets. The embedding in $h$-BN gave sufficient hydrostaticity to allow the activation volume determi- nation, giving insights on the conduction mechanisms. External heating of the piston-cylinder apparatus assured a simple cell configuration with low parasitic inductance, which allowed IS measurements over a broad range of frequencies. The temperature range covered allowed the determination of the activation energy in several systems. ${ }^{3-5}$ The activation volume of several ionic conductors was calculated from pressure dependence of bulk conductivity, derived from impedance spectra measured with the aid of this simple highpressure cell.

\section{ACKNOWLEDGMENTS}

The authors thank Professor G. Tomandl (Institut für Werkstoffwissenschaften, University of Erlangen-Nürnberg, Germany) for helpful discussions on impedance spectroscopy and Professor J. Vrbka (Department of Mechanics, TU of Brno, Czech Republic) for the dimensional calculations of the high-pressure compound cylinder.

${ }^{1}$ J. R. MacDonald, Impedance Spectroscopy, Emphasizing Solid Materials and Systems (Wiley, New York, 1987).

${ }^{2}$ G. A. Samara, Solid State Phys. 38, 1 (1984).

${ }^{3}$ R. Hinrichs, G. Tomandl, and J. A. H. da Jornada, Solid State Ion. 77, 257 (1995).

${ }^{4}$ R. Hinrichs, Ph.D. thesis, Erlangen, 1995.

${ }^{5}$ R. Hinrichs, A. Regis, A. Gruger, and Ph. Colomban, Synth. Met. 81, 227 (1996).

${ }^{6}$ F. R. Boyd and J. L. England, J. Geophys. Res. 65, 741 (1960).

${ }^{7}$ S. Lansiart and M. Beyeler, J. Phys. Chem. Solids 36, 703 (1975). 